\title{
Loss of Taste and Smell are Common Clinical Characteristics of Patients with COVID-19 in Somalia: A Retrospective Double Centre Study
}

This article was published in the following Dove Press journal: Infection and Drug Resistance

\section{Mohamed Farah Yusuf Mohamud (D) Yahye Garad Mohamed (D) Abdiladhif Mohamed Ali Bakar Ali Adam}

Mogadishu Somali-Turkish Training and Research Hospital, Mogadishu, Somalia
Purpose: The purpose of this study was to identify the common clinical presentations and the evidence on the presence of ageusia and anosmia as an emerging coronavirus disease 2019 (COVID-19) symptom to better inform in both COVID-19 patients and clinicians.

Patients and Methods: As part of a double-institutional collaboration coordinated by doctors, this study retrospectively collected and analyzed the clinical characteristics of 60 patients with COVID-19 pneumonia between April 1 and April 20, 2020. Pregnant women and patients taking anti-cancer drugs had been excluded from the study. Data from each institution's electronic medical record had been obtained.

Results: Sixty patients who had RT-PCR positive for COVID-19 were included in this study; of these patients, all of them had unknown exposure to COVID-19. The mean (SD) age was 45.7 (13.5) years, and 42 were men (70\%). Of these patients, $80 \%$ had at least ageusia or anosmia. The most common symptoms at the onset of illness were cough $(75 \%)$, fever $(71.3 \%)$, myalgia or fatigue (53.3\%), anosmia (loss of smell) (40\%), ageusia (loss of taste) $(28.3 \%)$, sore throat (25\%), shortness of breath (16.7\%), headache (16.7\%), and GI symptoms (diarrhea, nausea, vomiting and loss appetite) (16.7\%). A total of $68.3 \%$ of COVID-19 infected patients had reported either loss of taste or smell, and about $33.3 \%$ of them had only loss of smell, while $23.3 \%$ of them had impaired taste, and $11.7 \%$ of COVID-19 infected patients had both taste and smell loss.

Conclusion: During the epidemic period of SARS-CoV-2 infection, when presenting patients with ageusia and anosmia, physicians should consider COVID-19 pneumonia as a differential diagnosis to achieve early identification, avoid the delayed diagnosis, and prevention of transmission.

Keywords: anosmia, ageusia, severe acute respiratory syndrome coronavirus 2, SARS-CoV -2 , pneumonia

\section{Introduction}

In early December 2019, a novel coronavirus (2019-nCoV) identified as the causative agent associated with a cluster of cases of acute pneumonia in people at Wuhan, Hubei Province, uses next-generation sequencing in Wuhan Institute. ${ }^{1}$ On January 30, 2020, WHO stated that the outbreak of 2019-nCoV constitutes a public health emergency of international concern and declared COVID19 as a pandemic. ${ }^{2,3}$

Fever, cough, shortness of breath, myalgia, and fatigue were the common clinical manifestations of COVID-19 patients. ${ }^{4}$ Also, Professor Claire Hopkinson
Correspondence: Mohamed Farah Yusuf Mohamud

Turkish Training and Research Hospital, Thirty Street Alikamin, Wartanabada

District, Mogadishu, Somalia

Tel +252615591689

Email m.qadar59@gmail.com
Infection and Drug Resistance 2020:13 2631-2635

2631 
observed that ageusia and anosmia were classic symptoms of COVID-19 patients in the UK, France, the USA, and Italy. ${ }^{5}$ Anosmia has already reported as a clinical manifestation of COVID-19 and another coronavirus. ${ }^{6}$ Therefore, this is very likely due to the direct damage of the coronavirus on the olfactory and gustatory receptors. ${ }^{7}$

The incomplete or complete loss of smell and taste with or without other diseases had a wide differential diagnosis. ${ }^{8}$ Several scientific types of research have demonstrated that impaired taste and smell could be a common manifestation of Covid-19.9,10

By April 17, 2020, 116 confirmed cases were reported in Somalia with 2 (1.7\%) recovered cases and 5 (4.3\%) deaths. Till now, the reported cases were mostly among adults, with no pediatric cases reported. ${ }^{11}$ The clinical profiles of 2019-nCoV infection in children are unknown. So far, no studies in Somalia have described ageusia and anosmia, an emerging COVID-19 symptoms patient with COVID-19. We aim to summarize the common clinical presentations and the evidence on the presence of ageusia and anosmia as an emerging COVID-19 symptom, to better inform both COVID-19 patients and clinicians.

\section{Methods}

\section{Study Population, Setting, and Data Collection}

For this retrospective, double-center study, we reviewed 60 patients with real-time reverse transcriptase polymerase-chain-reaction (RT-PCR) confirmed Covid-19 pneumonia who had clinically presented to double hospitals in Mogadishu, Somalia, between April 1, 2020 to April 20, 2020. Only RT-PCR-confirmed cases included in this study. Pregnant women, patients taking anti-cancer drugs, and children (those younger than 18 years of age) were excluded from the study. Sixty adults (18 years of age or older) identified from two hospitals, including Mogadishu Somali Turkish Training and Research Hospital and Demartino Hospital. Data from each institution's electronic medical record was obtained. We obtained demographic data (age, sex, level of educational, and marital status); general clinical information (comorbidities and smoking status); and the signs and symptoms (fever, dry cough, impairment of taste and smell, shortness of breath, myalgia or fatigue, sore throat, headache, diarrhea, nausea, vomiting and impairment appetite).

\section{DATA Sources/Specimen Collection and Testing}

We performed a retrospective double centre study on the clinical records of 60 RT-PCR confirmed patients with COVID-19 that diagnosed from April 1 to April 20, 2020. The final follow-up for this report lasted until April 28, 2020.

Real-time reverse transcriptase polymerase-chainreaction (RT-PCR) assay test is the primary method of diagnosis to confirm COVID-19 by using throat swab specimens that collected from upper respiratory tracts following Centers for Disease Control and Prevention (CDC) guidelines. This test had done at different intervals. Of the 60 cases reported here, all cases RT-PCR tested were positive for SARS-CoV-2 once.

This retrospective study received medical ethical committee approval from the institutional review board of Mogadishu Somalia-Turkey Recep Tayyip Erdogan Training and Research Hospital, Mogadishu, Somalia (2020). In agreement with the declaration of Helsinki, the required patients written informed consent had waived by the ethical committee and data collected from medical records, confidential, no harm could potentially do to the patients and did not contain information that could identify individual personal information (anonymization).

\section{Statistical Analysis}

Descriptive statistics had used to summarize the data; results reported as medians and means and standard deviations, as appropriate. Categorical variables summarized as counts and percentages. No imputation had made for missing data. The analysis performed with IBM SPSS statistics version 23.0.

\section{Results}

Sixty patients who had RT-PCR positive for COVID-19 were included in this study; of these patients, all of them had unknown exposure to COVID-19. Their clinical characteristics had shown in (Table 1). The mean (SD) age was 45.7 (15.5) years, and 42 were men (70\%). Of these patients, $75 \%$ had at least ageusia or anosmia. The male gender remained the predominant cases, $60 \%$ had a loss of taste and smell compared with females. Comorbidities were present in $18.3 \%$ of patients, with diabetic being the most common comorbidity, followed by hypertension and chronic obstructive pulmonary disease (COPD) (Table 1). 
Table I Clinical Characteristics of 60 Adult Patients with COVID-I9 Pneumonia

\begin{tabular}{|c|c|c|}
\hline \multicolumn{3}{|c|}{ Patient Characteristics Patients $(n=60)$} \\
\hline \multicolumn{3}{|c|}{ Patient demographics } \\
\hline \multirow[t]{2}{*}{ Sex } & Male & $42(70 \%)$ \\
\hline & Female & $18(30 \%)$ \\
\hline \multirow[t]{3}{*}{ Age } & $19-39$ & $4 \mathrm{I}(68.3 \%)$ \\
\hline & $40-59$ & II (I8.3\%) \\
\hline & $\geq 60$ & $8(13.4 \%)$ \\
\hline \multirow[t]{4}{*}{ Marital status } & Single & $17(28.3 \%)$ \\
\hline & Married & $43(71.7 \%)$ \\
\hline & Divorced & $0(0 \%)$ \\
\hline & Widowed & $0(0 \%)$ \\
\hline \multirow[t]{5}{*}{ Level of education } & Illiterate & $4(6.7 \%)$ \\
\hline & Primary and intermediate & $3(5 \%)$ \\
\hline & school & \\
\hline & Secondary school & $4(6.7 \%)$ \\
\hline & Tertiary (University) & $49(81.7 \%)$ \\
\hline \multirow[t]{3}{*}{ Smoking status } & Non-smoker & $54(90 \%)$ \\
\hline & Past smoker & $6(10 \%)$ \\
\hline & Current smoker & $0(0 \%)$ \\
\hline \multirow[t]{2}{*}{ Exposure history } & Unknown exposure & $60(100 \%)$ \\
\hline & Exposure to positive patients & $0(0 \%)$ \\
\hline \multirow{3}{*}{$\begin{array}{l}\text { Comorbid } \\
\text { conditions }\end{array}$} & Diabetes & 7 (I I.7\%) \\
\hline & Hypertension & $3(5 \%)$ \\
\hline & COPD & I (I.7\%) \\
\hline \multicolumn{3}{|l|}{ Signs and symptoms } \\
\hline \multicolumn{2}{|l|}{ Dry cough } & 45 (75\%) \\
\hline \multicolumn{2}{|l|}{ Fever } & $43(71.7 \%)$ \\
\hline \multicolumn{2}{|l|}{ Myalgia or fatigue } & $32(53.3 \%)$ \\
\hline \multicolumn{2}{|c|}{ Anosmia (loss of smell) } & $24(40 \%)$ \\
\hline \multicolumn{2}{|c|}{ Ageusia (loss of taste) } & 17 (28.3\%) \\
\hline \multicolumn{2}{|c|}{ Sore throat } & $15(25 \%)$ \\
\hline \multicolumn{2}{|l|}{ Shortness of breath } & $10(16.7 \%)$ \\
\hline \multicolumn{2}{|l|}{ Headache } & $10(16.7 \%)$ \\
\hline \multicolumn{2}{|c|}{$\begin{array}{l}\text { GI Symptoms (diarrhea, nausea, vomiting and loss } \\
\text { appetite) }\end{array}$} & $10(16.7 \%)$ \\
\hline
\end{tabular}

Onset and recovery of anosmia and ageusia

\begin{tabular}{|l|l|l|}
\hline Onset of anosmia & Before the typical symptoms & $3(5 \%)$ \\
& With the typical symptoms & $6(10 \%)$ \\
& After the typical symptoms & $11(18.3 \%)$ \\
& Not remember & $4(6.7)$ \\
\hline Onset of ageusia & $\begin{array}{l}\text { Before the typical symptoms } \\
\text { With the typical symptoms }\end{array}$ & $2(3.4 \%)$ \\
& $\begin{array}{l}\text { After the typical symptoms } \\
\text { Not remember }\end{array}$ & $8(13.3 \%)$ \\
& Not & $3 \%)$ \\
\hline
\end{tabular}

(Continued)
Table I (Continued).

\begin{tabular}{|l|l|l|}
\hline \multicolumn{2}{|l|}{ Patient Characteristics Patients $(\mathbf{n}=60)$} \\
\hline Recovery of & $<5$ days & $15(25 \%)$ \\
anosmia & $5-10$ days & $3(5 \%)$ \\
& $>10$ days & $6(10 \%)$ \\
\hline \multirow{2}{*}{ Recovery of ageusia } & $<5$ days & $10(16.7 \%)$ \\
& $5-10$ days & $3(5 \%)$ \\
& $>10$ days & $4(6.6 \%)$ \\
\hline
\end{tabular}

The most common symptoms at the onset of illness were cough $(75 \%)$, fever $(71.3 \%)$, myalgia or fatigue (53.3\%), anosmia (loss of smell) (40\%), ageusia (loss of taste) $(28.3 \%)$, sore throat $(25 \%)$, shortness of breath (16.7\%), headache (16.7\%), and GI symptoms (diarrhea, nausea, vomiting and impaired appetite) (16.7\%).

A total of $68.3 \%$ of COVID-19 infected patients had reported either impaired of taste or smell, about $33.3 \%$ of them had an only loss of smell, while about $23.3 \%$ of them had an impaired taste only, and $11.7 \%$ of COVID-19 infected patients had both taste and smell impaired.

Patients who exhibited impaired smell were four groups, about $5 \%$ developed before typical symptoms of COVID-19, while about $18.3 \%$ manifested impaired smell after typical symptoms, and $10 \%$ develop at the same time, while $6.7 \%$ of patients did not remember the specific time of onset. Moreover, patients who had developed impaired taste before typical symptoms of COVID-19 were about $3.4 \%$, while $13.3 \%$ developed after typical symptoms, about $6.6 \%$ develop at the same time, and $5 \%$ of patients did not remember the time of onset.

Our search identified that about 25\% of COVID-19 infected patients had recovered from the loss of smell for less than five days. While about $5 \%$ had recovered within $5-10$ days and about $10 \%$ of these patients were unrecovered for more than 10 days. Moreover, about $16.7 \%$ of COVID-19 infected patients had recovered from the loss of taste in less than 5 days. While about $5 \%$ had recovered within 5-10 days, and about $6.6 \%$ of these patients were unrecovered for more than 10 days.

Moreover, there was no association between comorbidities and the development of loss of taste or smell in severe acute respiratory syndrome coronavirus 2 (SARS-CoV-2), and this indicated that only one patient who had diabetes developed an impaired taste. 


\section{Discussion}

The clinical characteristics of 60 patients with the laboratory-confirmed novel coronavirus (2019-nCoV) from two selected hospitals in Mogadishu, Somalia, between April 1 to April 20, 2020, were included in this study.

Seventy five percent of the patients with or without typical symptoms (fever, cough, and fatigue or myalgia) of COVID19 came to the hospital with impaired taste and the smell as their presenting features in contrast to previous studies. ${ }^{12-14}$

Taste and smelling disorders are well known to be related to a wide range of viral infections. ${ }^{15}$ A research study in Wuhan recently published on the neurologic manifestations of 214 SARS-CoV-2-positive hospitalized patients. The study showed that $11(5.1 \%)$ patients and ageusia in $12(5.6 \%)$ patients. ${ }^{16}$ In our study, impaired taste and smell were among the two main clinical findings in patients with COVID-19 infection in the absence or presence of other symptoms similar to the previous studies that about $85.6 \%$ of patients reported an isolated loss of taste, $79.6 \%$ of them having loss of smell, and $55 \%$ had combined loss of taste and smell. ${ }^{17,18}$

A cross-sectional research study in Milan in 59 SARS-CoV -2-positive hospitalized patients had disturbances of smell and taste. The study showed that $34 \%$ of the patients had a loss of smell and taste, $20 \%$ before, and $14 \%$ after hospitalization. ${ }^{19}$

Patients who exhibited impaired smell were four groups, about $5 \%$ developed before typical symptoms of COVID-19, while about $18.3 \%$ manifested impaired smell after typical symptoms, and $10 \%$ develop at the same time, while $6.7 \%$ of patients did not remember the specific time of onset. Moreover, patients who had developed impaired taste before typical symptoms of COVID-19 were about $3.4 \%$, while $13.3 \%$ developed after typical symptoms, about $6.6 \%$ develop at the same time, and $5 \%$ of patients did not remember the time of onset.

In our study, loss of taste and smell was prevalent in patients with mild-to-moderate SARS-CoV-2 infection that was consistent with the previous studies. ${ }^{20}$

The limitations of the present study are: first, it was a retrospective study design, and the number of cases was small, including only 60 patients. Second, all data collected from the electronic medical records; some patients with impairment taste and smell might not be involved in the study due to too mild impairment. Finally, in our research, most of the symptoms were a patient's subjective descriptions. We could not distinguish whether impaired of taste and smell caused by the COVID-19 virus directly or by the neurological disease or other infections or other organ damage indirectly.
However, by including all adult patients in the two designated hospitals for COVID-19, we believe our study population is representative of cases of experienced disturbance of taste and smell in Mogadishu. According to knowledge, this is one of the most significant retrospective studies among patients with COVID-19 who have experienced impaired taste and smell.

In conclusion, during the epidemic period of SARSCoV-2 infection, the patients presenting with ageusia and anosmia, physicians should consider COVID-19 pneumonia as a differential diagnosis to achieve early identification, avoid the delayed diagnosis, and prevention of transmission. Also, impaired smell and taste in COVID19 infected patients would allow self-isolation and urge communication to their healthcare providers.

We recommend conducting further research to provide valid and reliable ways to achieve early identification and avoid the delayed diagnosis of this public health emergency.

\section{Disclosure}

The authors report no conflicts of interest in this work.

\section{References}

1. Zhou P, Yang XL, Wang XG, et al. A pneumonia outbreak associated with a new coronavirus of probable bat origin. Nature. 2020;579 (7798):270-273.

2. World Health Organization. Statement on the second meeting of the International Health Regulations (2005) Emergency Committee regarding the outbreak of novel coronavirus (2019-nCoV).

3. WHO. WHO Director-General's opening remarks at the media briefing on COVID-19-11 March 2020. 2020. Available from: https:// www. who.int/dg/speeches/detail/who-directorgeneral-s-openingremarks-at-the-mediabriefing-on-covid-19-11-march-2020. accessed March 11, 2020.

4. Chen N, Zhou M, Dong X, et al. Epidemiological and clinical characteristics of 99 cases of 2019 novel coronavirus pneumonia in Wuhan, China: a descriptive study. The Lancet. 2020;395 (10223):507-513. doi:10.1016/S0140-6736(20)30211-7

5. Hopkins C, Kumar N. Loss of sense of smell as marker of COVID-19 infection. ENT UK. 2020;26(03):2020.

6. de Haro-licer J, Roura-Moreno J, Vizitiu A, González-Fernández A, González-Ares JA. Long term serious olfactory loss in colds and/or flu. Acta Otorhinolaryngologica (English Edition). 2013;64 (5):331-338. doi:10.1016/j.otoeng.2013.10.004

7. Yamagishi M, Fujiwara M, Nakamura H. Olfactory mucosal findings and clinical course in patients with olfactory disorders following upper respiratory viral infection. Rhinology. 1994;32(3):113-118.

8. Doty RL. Epidemiology of smell and taste dysfunction. In: Handbook of Clinical Neurology. Vol. 164. Elsevier; 2019:3-13.

9. Wee LE, Chan YF, Teo NW, et al. The role of self-reported olfactory and gustatory dysfunction as a screening criterion for suspected COVID-19. Eur Arch Oto-Rhino-Laryngol. 2020;1-2.

10. Printza A, Constantinidis J. The role of self-reported smell and taste disorders in suspected COVID-19. Eur Arch Oto-Rhino-Laryngol. 2020;1-6. 
11. SONNA. Dowladda Oo Xaqiijisay in ay sooKordheenXaaladoCusub oo COVID-19 Ah, Somalia. 2020. Available from: https://sonna.so/ dowladda-oo-xaqiijisay-in-ay-soo-kordheen-xaalado-cusub-oo-covid -19-ah/. Accessed April 17, 2020.

12. Lovato A, de Filippis C. Clinical presentation of COVID-19: a systematic review focusing on upper airway symptoms. Ear Nose Throat J. 2020;0145561320920762.

13. Wang P, Lu JA, Jin YY Epidemiological characteristics of 1212 COVID-19 patients in Henan. China. 2020.

14. Beltrán-Corbellini Á, Chico-García JL, Martínez-Poles J, et al. Acute-onset smell and taste disorders in the context of COVID-19: a pilot multicentre polymerase chain reaction based case-control study. Eur J Neurol. 2020;22.

15. van Riel D, Verdijk R, Kuiken T. The olfactory nerve: a shortcut for influenza and other viral diseases into the central nervous system. J Pathol. 2015;235(2):277-287. doi:10.1002/path.4461
16. Mao L, Jin H, Wang M, et al. Neurologic manifestations of hospitalized patients with coronavirus disease 2019 in Wuhan, China. JAMA Neurol. 2020;77(6):683. doi:10.1001/jamaneurol.2020.1127

17. Vaira LA, Hopkins C, Salzano G, et al. Olfactory and gustatory function impairment in COVID-19 patients: italian objective multicenter-study. Head Neck. 2020;42(7):1560-1569. doi:10.1002/hed.26269

18. Cabaraux P, Lechien JR, Chiesa-Estomba CM, et al. Objective olfactory evaluation of self-reported olfactory dysfunction in a case series of 86 COVID-19 patients. medRxiv. 2020.

19. Giacomelli A, Pezzati L, Conti F, et al. Self-reported olfactory and taste disorders in patients with severe acute respiratory coronavirus 2 infection: a cross-sectional study. Clin Infect Dis. 2020. doi:10.1093/cid/ciaa330

20. Lechien JR, Chiesa-Estomba CM, De Siati DR, et al. Olfactory and gustatory dysfunctions as a clinical presentation of mild-to-moderate forms of the coronavirus disease (COVID-19): a multicenter European study. Eur Arch Oto-Rhino-Laryngol. 2020;6:1.
Infection and Drug Resistance

\section{Publish your work in this journal}

Infection and Drug Resistance is an international, peer-reviewed openaccess journal that focuses on the optimal treatment of infection (bacterial, fungal and viral) and the development and institution of preventive strategies to minimize the development and spread of resistance. The journal is specifically concerned with the epidemiology of
Dovepress

antibiotic resistance and the mechanisms of resistance development and diffusion in both hospitals and the community. The manuscript management system is completely online and includes a very quick and fair peerreview system, which is all easy to use. Visit http://www.dovepress.com/ testimonials.php to read real quotes from published authors. 